\title{
Application of fractal algorithms of coastline echo's generation on marine radar simulator
}

\author{
Shuguang $\mathrm{Ji}^{1 *}$, Zhang Zhang ${ }^{2}$, Hongbiao Yang ${ }^{1}$, Dan Liu³ and Rapinder Sawhney ${ }^{1}$
}

\begin{abstract}
Background: Marine radar simulator is a useful approach endorsed by International Maritime Organization (IMO) to train the seafarers on how to operate marine radar equipment and use marine radar equipment for positioning and collision avoidance in laboratory. To fulfill all of the marine radar simulator training requirements, a high performance simulator is necessary. However, imperfections with currently available marine radar simulators require simulator developers to make improvements.

Case description: In this study, improved fractal algorithms (random Koch curve, fractional Brownian motion, and Weierstrass-Mandelbrot function) are applied to generate natural-looking radar echoes on a marine radar simulator.

Discussion and evaluation: From the results of the simulations, we can observe that the structures of the coastline echoes generated by improved fractal algorithms, especially by fractional Brownian motion algorithm, outperform the echoes generated by conventional method in representing a natural coastline feature.

Conclusions: Based on evaluations from a panel of experienced mariners, we conclude that the coastline echoes simulated by fractal algorithms better represent a natural coastline feature than those generated by conventional methods.
\end{abstract}

Keywords: Marine radar simulator, Coastline echo, Fractal algorithm

\section{Background}

The International Convention on Standards of Training, Certification and Watchkeeping for Seafarers 78/95 (STCW Convention 78/95) of International Maritime Organization (IMO) requires using a marine radar simulator to train seafarers. A marine radar simulator is the only acceptable approach in the laboratory for seafarers to learn how to operate radar equipment and use radar for positioning, navigation, and anti-collision. Upon completing all requirements set forth in the training, the trainee will receive certification for qualifications to work on board. Currently, marine radar simulators are widely used by the members of IMO as one of many useful tools for seafarer education and training (Ali 2006; Organization 2006; Teel et al. 2009; Xiuwen et al. 2010). However,

*Correspondence: sji1@utk.edu

1 University of Tennessee, 37996 Knoxville TN, USA

Full list of author information is available at the end of the article due to the limitation of the simulation technology, the marine radar simulators on the market are unable to replicate the performance of real radar equipment. Taking the simulation of coastline echoes as an example, the coastline echo is generated by raw data, which are acquired from the digitalized chart and consist of a collection of coordinate points. By connecting two adjacent coordinate points, a straight line is generated to approximate a real coastline echo. This method works well for generating a coastline echo under a large radar range (say 6 nautical miles (NM)). However, when the radar range is adjusted to a smaller radar range (such as $0.25 \mathrm{NM}$ ), the shape of coastline echo will lose its natural structure and look quite artificial (Ji et al. 2015; Zhang 2007). In addition, it should take around three seconds for the scan line of the marine radar to rotate a round. By adopting a traditional generation method for the coastline echo under small radar 
range, the time for the scan line to rotate around is much more likely to exceed three seconds, since extra sampling coordinate points (if available) have to be inserted to generate a high quality coastline echo. Imperfections like the artificial coastline shape and slow rotation of the radar scan line may have negative impacts on the training effectiveness for users (Ji et al. 2015; Zhang 2007). In order to overcome the problems associated with the conventional coastline echo simulation approach, we apply fractal theory to the coastline echo simulation process, since fractal theory is widely used as a graphics tool for generating natural-looking shapes like coastlines, rivers, mountains, and other natural features (Pentland 1984). The simulation results are evaluated and scored by 30 experienced mariners to validate that the coastline echoes generated by fractal algorithms look more natural than those generated by conventional method. Furthermore, an improved fractal algorithm is designed to guarantee the scan line can finish a round of rotation within three seconds, which is difficult to be achieved using the conventional method, especially under a larger radar range.

\section{Literature review}

The STCW Convention provides required components for seafarer training, which use the radar simulator as a tool of training and assessment. These highlights include (Ali 2006; Organization 2006; Teel et al. 2009): factors affecting performance and accuracy; detection of misrepresentation of information, including false echoes and sea returns; setting up and maintaining displays; range and bearing; plotting techniques and relative motion concepts; identification of critical echoes; course and speed of other ships; time and distance of the closest approach to crossing, meeting or overtaking ships; detecting course and speed changes of other ships; effects on the changes of the own ship's course or speed or both; and application of the International Regulations for Preventing Collisions at Sea. To fulfill all of these training requirements, a high performance marine radar simulator is needed. In the current marine radar simulator market, the major developing teams include Nautical Software (2016), Bridge Command (2016), Kongsberg Maritime (2016), Landfall (2016), and Dalian Maritime University Institute of Navigational Technology (2016). In addition, some previous research investigates methods to improve the marine radar simulator. For example, Arnold-Bos et al. developed a versatile bistatic and polarimetric marine radar simulator. In their simulator, realistic sea surfaces are generated using the two-scale model on a semi-deterministic basis, so as to incorporate the presence of ship wakes in the simulation (Arnold-Bos et al. 2006). Yin et al. designed a radar simulator using a PC to generate radar echoes and a radar interface board to generate radar signals. Their simulator has a more flexible and realistic operation interface than other simulators (Yin et al. 2007). Zhang et al. put forward a coastline echo intensity algorithm based on RGB and HIS color models and applied this algorithm on the marine radar simulator. The simulation results from this model are consistent with the electronic chart (Zhang et al. 2010).

In this study, we incorporate fractal theory, a branch of non-linear mathematics, to improve coastline echo simulation. The research targets of fractal theory are irregular objects and non-linear systems in the nature. The term "fractal"? was first used by mathematician Benoit Mandelbrot in 1975 to extend the concept of theoretical fractional dimensions to geometric patterns in nature (Mandelbrot 1983). In the 1980s, fractal theory was applied into the signal processing for radar because the echoes reflected into radar system have many fractal patterns (Ji et al. 2015; Zhang 2007). Even though fractal theory has been widely applied in fields such as virtual reality, image processing, and time series analysis, etc. (Ji et al. 2005; Zhang et al. 2005), there are few studies to apply it into the simulation of coastline echo for marine radar simulator. This research aims to close this gap. Partial of findings reported in this article were originally presented at the 94th Transportation Research Board Annual Meeting. We improved the research methodology in Ji et al. (2015) in this article. Especially, a full control of the physical parameters involved fractal function, Weierstrass-Mandelbrot function (WMF), is used to simulate coastline echoes. In addition, a quantitative validation of the simulation results is designed to assess the fidelity of the simulation outcomes and comprehend possible values of the introduced parameters among the simulation algorithms.

\section{Methods}

The echo reflection on radar simulator can be classified into three types (Ji et al. 2015; Zhang 2007). A Type I Echo is the echo reflected by artificial architectures such as berth and breakwater. Type I Echoes have regular shape and can be used for positioning because of its clear boundaries and fixed position. A Type II Echo is the echo reflected by rocky coast. Type II Echoes have a realistic pattern as well as fixed position. A Type III Echo is an echo reflected by flat coast such as sand coast. Type III Echoes have a large echo reflection zone and relatively weak reflection. Additionally, the shape and position of a Type III Echo will change with the motion of the waves. This study focuses only on simulation of Type II Coastline Echoes because of its natural fractal features. Three different fractal algorithms are adopted to simulate coastline echoes. A comparison among these three simulation algorithms is included. 


\section{Random Koch curve algorithm}

The Koch curve was described by Swedish mathematician Helge von Koch in 1904 (Keddam and Takenouti 1988). The curve can be constructed as shown in Fig. 1. First, a straight line is divided into tree equal segments, and an equilateral triangle is raised from the middle third. The resulting line segments are again divided into thirds with an equilateral triangle raised from the middle third of each. This process is repeated through the desired number of iterations (Cross 1994). The length of the Koch curve can be infinite because the total length of the curve increases by one third with each iteration (Baliarda et al. 2000). The dimension of Koch curve is 1.262 . This makes us consider Koch curve a candidate to simulate coastlines, since coastlines are fractal curves with dimensions ranging from 1 (very smooth) to 1.5 (very rough)(Aviles and Scholz 1987). From Fig. 1 we can see that the resulting curve is roughly similar to the natural pattern of a coastline.

However, the Koch curve generated through this theoretical approach is too regular and symmetric to simulate a coastline echo, since the inlets in real coastlines are similar to each other but not identical. Thus, we incorporate randomness into the regular Koch curve generation process to produce a more natural-looking coastline echo (Ji et al. 2015; Zhang 2007). In order to add randomness into the Koch curve, half of the time, we raise the equilateral triangle from the middle third; half of the time, we bent the equilateral triangle down from the middle third (Filoche and Sapoval 2000). In Fig. 2, Koch curves generated by regular and random methods are compared. When comparing to a naturally forming coastline, the

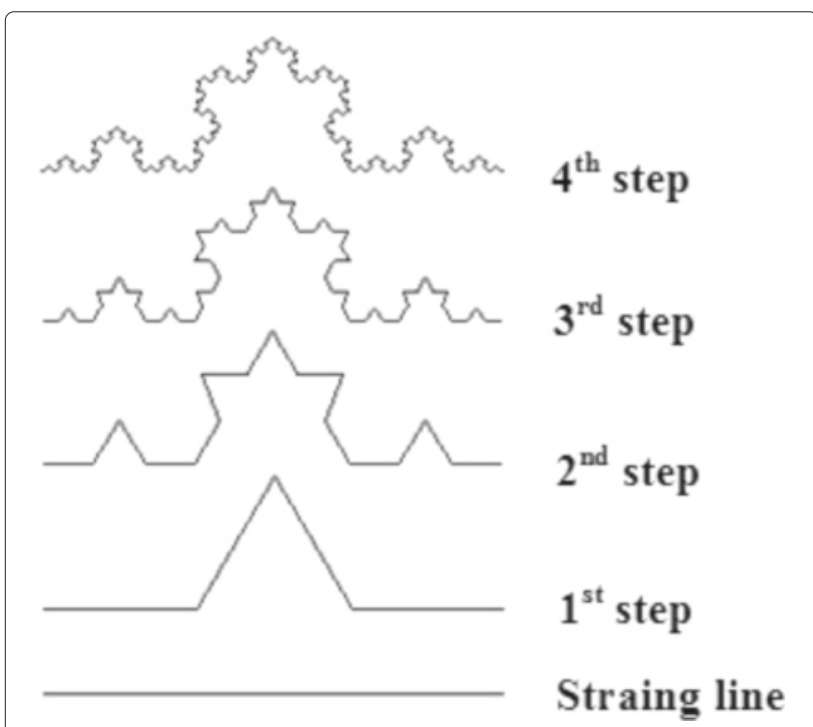

Fig. 1 Construction of regular Koch curve

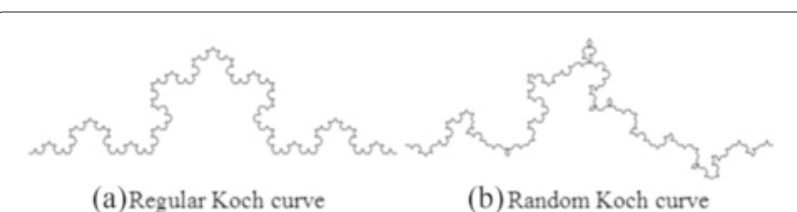

Fig. 2 Comparison between regular and random Koch curve

random Koch curve looks more realistic than the regular Koch curve, as the regular Koch curve appears too artificial to exist in a natural environment.

Even though the random Koch curve is an improved method for coastline echo simulation, application of this method without constraints may bring distortion into the simulation process. As long as the distance between two adjacent sample points is short, the distortion from the simulation of the coastline echo is negligible. However, as the distance between sample points increases, the distortion becomes more noticeable. For example, as shown in Fig. 3, the trend of the simulated coastline echo in the two boxes (shown in Fig. 3b) is different from the original trend of coastline echo (shown in Fig. 3a) (Ji et al. 2015; Zhang 2007).

To avoid the above distortion, a control value (c) is introduced into the simulation process (in this study, $\mathrm{c}=15$ ). Once the distance between two adjacent sample points is shorter than c, the random Koch curve is used directly to simulate the coastline echo between these two adjacent sample points. If this distance is larger than c, several random points are inserted between the sample points to break the original line into smaller segments. Then, the random Koch curve algorithm is applied between new adjacent sample points to simulate the coastline echo. The value $\mathrm{c}$ can be adjusted in accordance with computer's performance. We can apply a smaller c value into the random Koch curve algorithm on a high performance computer. As shown in Fig. 4, after adopting a random Koch curve algorithm with a control value, the distortion of the trend

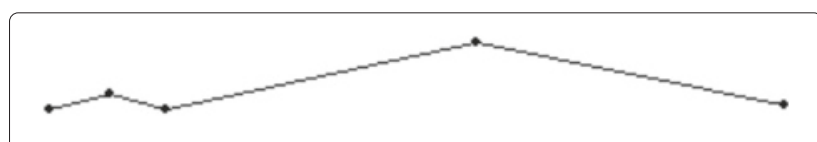

(a) Original trend of coastline's echo

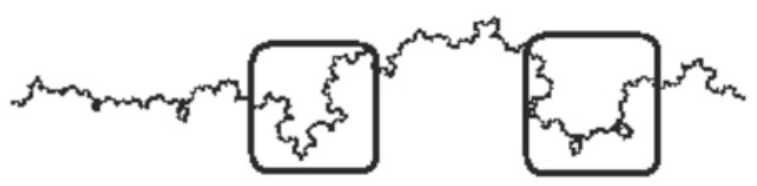

(b) Trend distortion of coastline's echo

Fig. 3 Trend distortion from random Koch curve algorithm 


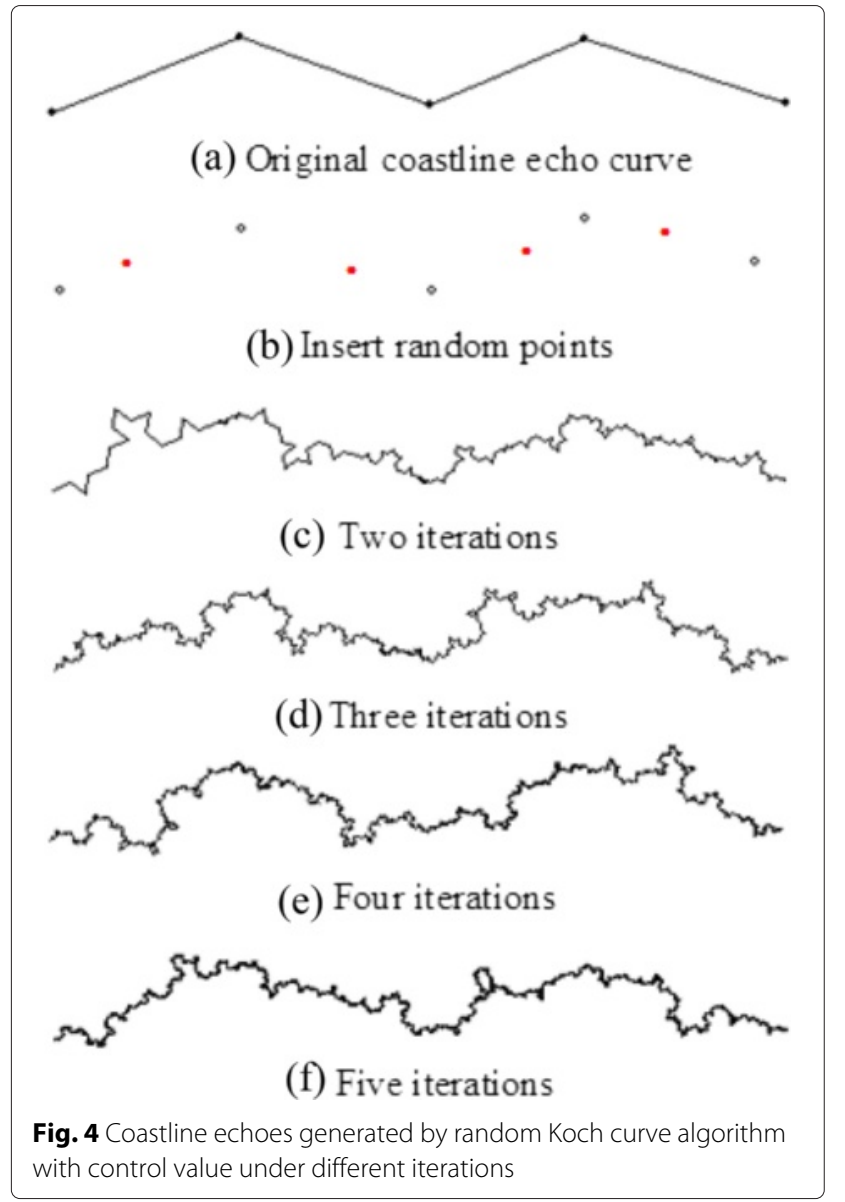

of simulated coastline echo is negligible. Also, we notice that there is no obvious improvement between the simulated coastline echoes if the iteration is more than three times based on visual inspection. Therefore, we suggest that three iterations are sufficient for the simulation of the coastline echo by random Koch curve algorithm with control value.

\section{Fractional Brownian Motion algorithm}

Fractional Brownian Motion (FBM) was introduced by Mandelbrot and van Ness in 1968 (Rostek and Schöbel 2013). We know that Brownian motion, which is also called Wiener process, is a random process (Falconer 2013). If $-\infty<t<\infty$ and $w$ belongs to a set of values of a random function and if the intervals $\left(t_{1}, t_{2}\right)$ and $\left(t_{3}, t_{4}\right)$ do not overlap, the ordinary Brownian motion $B(t, w)$ is a real random function with independent Gaussian increments. Therefore, $B\left(t_{2}, w\right)-B\left(t_{1}, w\right)$ has a mean of zero and a variance $\left|t_{2}-t_{1}\right|$. Also, $B\left(t_{2}, w\right)-B\left(t_{1}, w\right)$ is independent of $B\left(t_{4}, w\right)-B\left(t_{3}, w\right)$. Let $0<H<1$ and $b_{0}$ be an arbitrary real number. For $t>0$, the random function $B_{H}(t, w)$ below is called a reduced FBM with Hurst coefficient $H$ and starting value $b_{0}$ at time 0 (Dieker 2004; Mandelbrot and Van Ness 1968).

$$
\begin{aligned}
& B_{H}(t, w)-B_{H}(0, w)=\frac{1}{\Gamma\left(H+\frac{1}{2}\right)}\left(\int _ { - \infty } ^ { 0 } \left[(t-s)^{H-\frac{1}{2}}-\right.\right. \\
& \left.\left.(-s)^{H-\frac{1}{2}}\right] d B(s, w)+\int_{0}^{t}(t-s)^{H-\frac{1}{2}} d B(s, w)\right)
\end{aligned}
$$

where $\Gamma$ represents the Gamma function: $\Gamma(\alpha)=$ $\int_{0}^{\infty} x^{\alpha-1} e^{-x} d x$ and $B_{H}(0, w)=b_{0}$ (Mandelbrot and Van Ness 1968).

In practice, the random midpoint displacement (RMD) method is used to simulate FBM. By the RMD method, the height of a midpoint between two other points is given by:

$$
h_{\text {new }}=\frac{1}{2}\left(h_{\text {left }}+h_{\text {right }}\right)+h_{\text {random }}
$$

where $h_{\text {random }}$ is a random offset. In our simulation, the coordinates of the midpoints are calculated by the formula below (Boyle et al. 2007):

$$
\begin{aligned}
& x_{i}^{\text {new }}=\frac{1}{2}\left(x_{i}+x_{i+1}\right)+h_{i} \\
& y_{i}^{\text {new }}=\frac{1}{2}\left(y_{i}+y_{i+1}\right)+h_{i} \\
& h_{i}=\Delta_{i} \cdot \operatorname{Gauss}(\cdot)=\frac{\sigma}{\left(2^{2 i}\right)^{H}} \sqrt{1-2^{2 H-2}} \cdot \operatorname{Gauss}(\cdot)
\end{aligned}
$$

where $\sigma$ is the standard deviation of the heights of sample points. $H$ is Hurst index and Gauss(.) is a random number generated by standard normal distribution which has a mean of zero and a standard deviation of one. The curves generated by the RMD method under different Hurst coefficients are shown in Fig. 5. The dimension of a

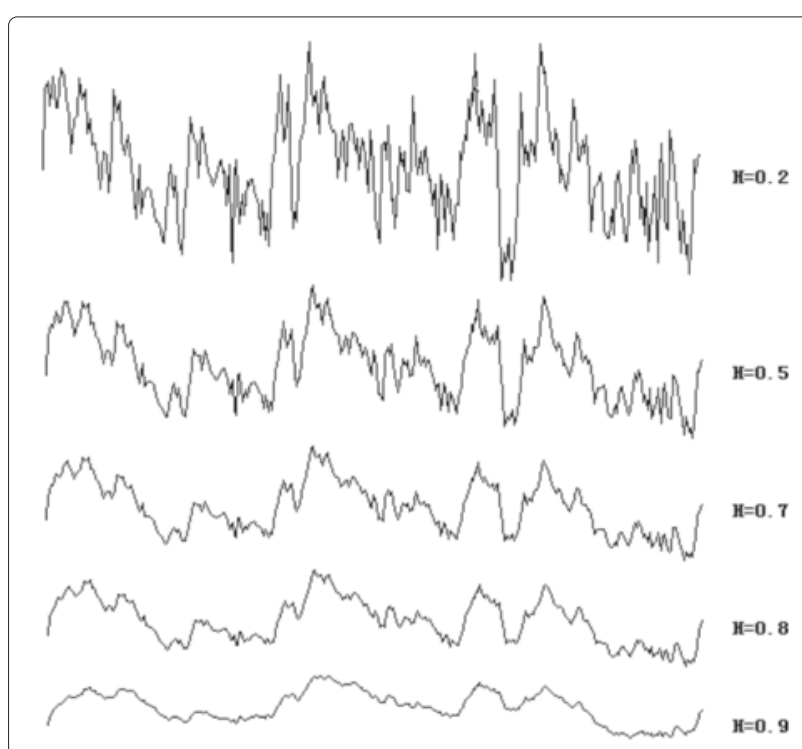

Fig. 5 The curves generated by FBM algorithm with different Hurst coefficient 
fractal curve generated by RMD method equals to $2-H$ (Huang et al. 1992). As we mentioned above, the dimensions of coastlines range from 1 to 1.5 . Therefore, we can select Hurt coefficient in the range of 0.5 and 1 to simulate coastline by RMD method. In this study, Hurst coefficient is set to 0.6 .

Figure $6 \mathrm{a}$ is the coastline echo generated by the conventional method. The results shown in the other subfigures of Fig. 6 are the coastline echoes generated by FBM method $(H=0.6)$ under a different number of iterations. It can be seen that the FBM method works better than the conventional method in terms of coastline echo simulation since more details are provided and the shape is approximate to a real coastline. In addition, we notice that there are no obvious differences between the coastline echoes with more than three iterations via visual inspection. We suggest that three iterations are sufficient for coastline echo simulation by the FBM method as well.

\section{Weierstrass-Mandelbrot function algorithm}

In 1872, German mathematician Karl Weierstrass devised a function, that is continuous everywhere but differentiable nowhere. This function is called Weierstrass function. The Weierstrass function is defined as:

$$
f(t)=\sum_{n=0}^{\infty} a^{n} \cos \left(b^{n} \pi t\right)
$$

where $0<a<1, b$ is a positive odd integer, and $a b>1+$ $1.5 \pi$ (Weierstrass 1967; Zhang et al. 2015). In 1977, Mandelbrot extended the Weierstrass function to the following form,which is called Weierstrass-Mandelbrot function. He also pointed out that the WMF is a fractal and has no

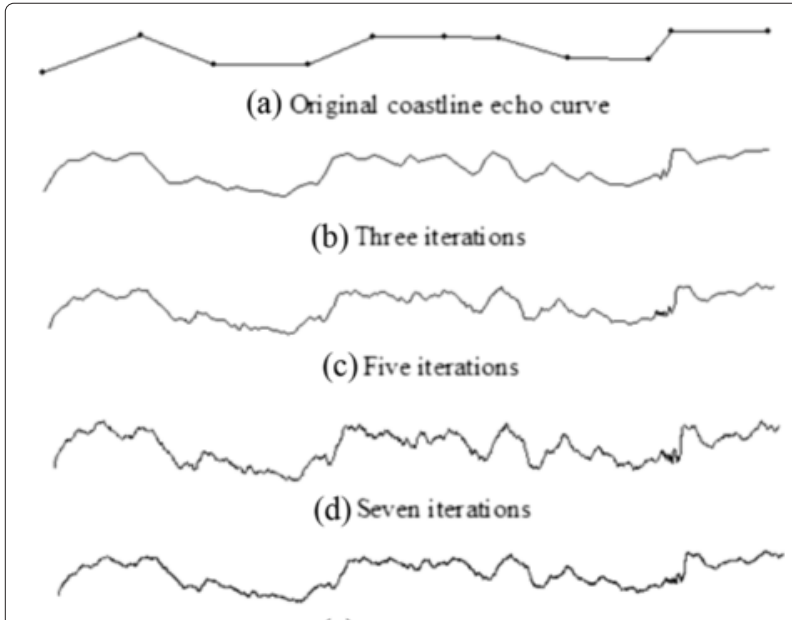

(e) Nine iterations

Fig. 6 Coastline echoes generated by FBM algorithm under different iterations $(H=0.6)$ smallest scale (Berry and Lewis 1980; Mandelbrot 1979). WMF has been widely adopted to simulate various phenomena in real world (Harrouni 2008; Ma et al. 2015; Shanhua et al. 2015; Wang et al. 2015; Zhang et al. 2015).

$$
W(t)=\sum_{n=-\infty}^{+\infty} \frac{\left(1-e^{i \gamma^{n} t}\right) e^{i \phi_{n}}}{\gamma^{(2-D) n}}
$$

where $D$ is the fractal dimension of the graph of $W(t)$ and $1<D<2 . \gamma$ is a parameter larger than 1 . $\phi_{n}$ is an arbitrary phase. When $\phi_{n}=0$, the form of WMF is:

$$
W(t)=\sum_{n=-\infty}^{+\infty} \frac{1-\cos \left(\gamma^{n} t\right)}{\gamma^{(2-D) n}}
$$

When $\phi_{n}=n \pi$, the form of WMF becomes:

$$
W(t)=\sum_{n=-\infty}^{+\infty} \frac{(-1)^{n} \sin \left(\gamma^{n} t\right)}{\gamma^{(2-D) n}}
$$

Without loss of generality, we use $\phi_{n}=0$ to generate cosine series to simulate the coastline echo (Zhang et al. 2015). By inspecting the simulation results under various combinations of $D$ and $\gamma$, we notice that the fractal dimension $D$ has much larger impact on the fluctuation frequency of the cosine series than parameter $\gamma$. Additionally, in order to keep consistent with the fractal dimension of actual signals, parameter $D$ should be selected between 1 and 1.5, since the fractal dimensions of actual signals range from 1 to 1.5 . Therefore, in this study, $\gamma$ and $D$ are set to 1.5 for both. Figure 7 presents the cosine series simulated in Matlab using aforementioned parameters. It can be seen that the cosine series generated by WMF have infinitely complex patterns across different scales. Based on the characteristics and previous applications of fractal theory (Majumdar and Tien 1990; Voss 1988; Yang et al.), the cosine series generated by WMF can be used to simulate the natural pattern of a coastline.

\section{Algorithm improvement}

We know that, in the process of simulating coastline echoes using fractal algorithms, a collection of sample or random points will be added in each iteration. As the number of added points increase, the simulation software will consume more computer resources, such as calculation ability and storage space, which may slow down the rotation of scan line, or even result in a buffer overflow. 

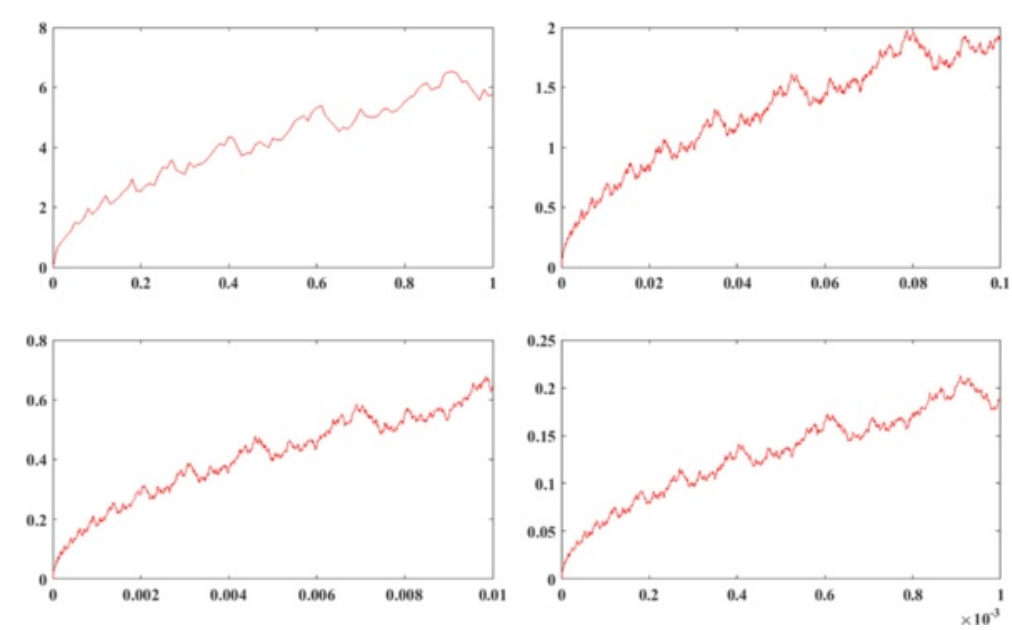

Fig. 7 Cosine series generated by WMF in different scales $\left(D=1.5, \gamma=1.5, \phi_{n}=0\right)$

As the radar range increases, the amount of information included in the radar simulation also increases. Typically, increasing iterations and radar range produce a slower rotating scan line or can even result in failure of the simulation. To troubleshoot this issue, we introduce a threshold value $(\mathrm{L})$ into the simulation process. Only when the distance between two adjacent sample points is larger than the threshold value $\mathrm{L}$ (in this study $L=10$ ), the fractal algorithms will be applied. Otherwise, the conventional simulation method will be used to generate the coastline echo. This is because the conventional simulation method works well enough under large radar ranges. Again, the value $\mathrm{L}$ can be adjusted in accordance with computer's performance. We can set $\mathrm{L}$ to a smaller value or even zero in the improved algorithm on a high performance computer. For those who are using similar computers as the authors do, $L=10$ is a good starting point. Figure 8 illustrates the flow chart of improved fractal algorithms for the generation of coastline echo.

\section{Result and discussion}

\section{Simulation outcomes on marine radar simulator}

The simulation algorithms were implemented in the $\mathrm{C}++$ language and tested on a laptop with an Intel(R) Core (TM) i5-2520M CPU, $2.50 \mathrm{GHz}$ processor, 2 cores, Intel HD Graphics 3000 graphic card, and 8 GB RAM (Windows 7 64-bit). Figure 9 presents a panel of simulation outcomes of the coastline echoes under $0.25 \mathrm{NM}$ radar range using the conventional method and fractal algorithms. All of the fractal algorithms uses three iterations. Figure 9a shows the coastline echoes generated by conventional method. It can be observed that the simulated coastline echoes by this method is in a regular straight line pattern without the natural features of coastline echoes.
In Fig. 9b, throw in some randomness, we notice that the regularity of simulate coastline echoes starts to fade out. While using FBM method to generate coastline echoes (as shown in Fig. 9c), the pattern of simulated coastline echoes looks interestingly real. The features of real coastline echoes, such as inlets and promontories can be observed clearly. The coastline echoes simulated by WMF method (as shown in Fig. 9d) show natural features of coastline echoes as well; however, the natural features of simulated coastline echoes by WMF method are not as obvious as those generated by FBM method. In summary, via visual inspection, we can observe that the structures of the coastline echoes generated by fractal algorithms (Fig. 9b, c, d) outperform the echo generated by conventional method in representing a natural coastline feature (Fig. 9a).

To support above conclusion from visual inspection, a survey is designed for a panel of 30 experienced mariners to collect their opinion on the patterns of simulated coastline echoes by conventional method and fractal algorithms. These mariners consist of 17 licensed officers on board (including two captains, two chief mates, three second mates, and ten third mates), five maritime enforcement investigators, and eight researchers in nautical science. The average working years of these mariners are 5.6 years ranging from 1 to 30 years. All the mariners report that they have experience of using radar equipment for positioning and navigation. The coastline echoes generated by different methods are presented to them. They are required to evaluate and score these coastline echoes. To be specific, in the first part of the survey, we provide the mariners three pairs of comparison figures of simulated coastline echoes (conventional method v.s. random Koch curve, conventional method v.s. FBM, and 


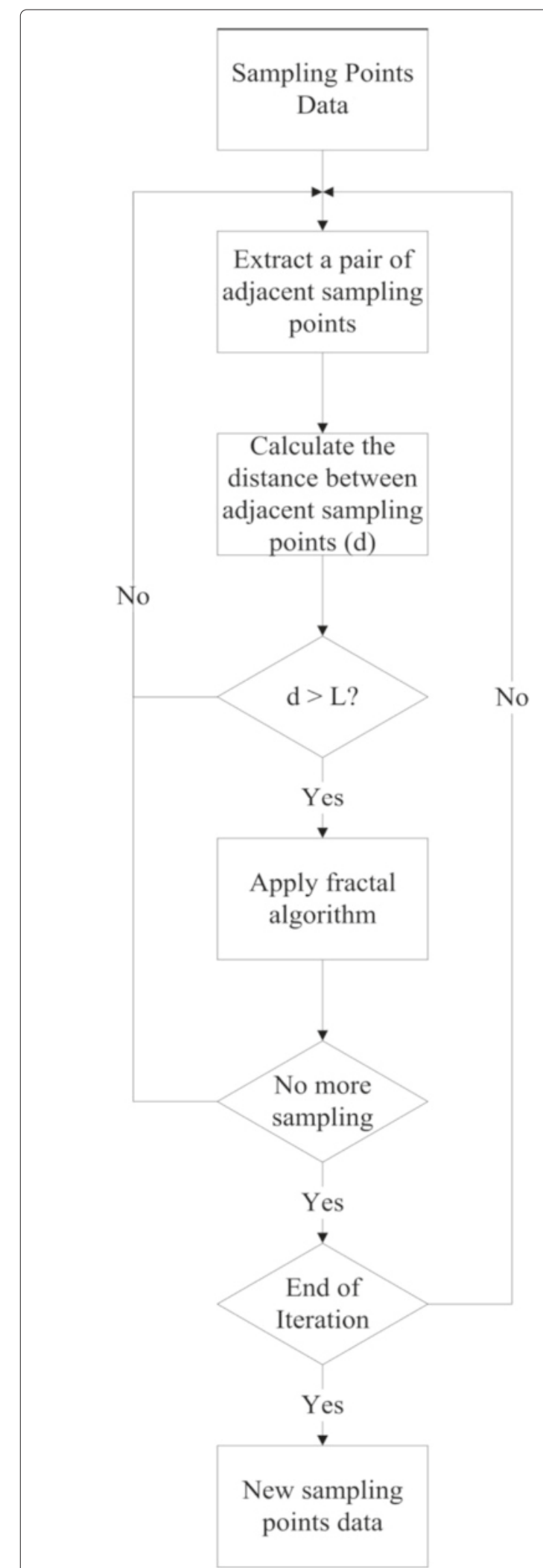

Fig. 8 Flow chart of improved fractal algorithm conventional method v.s. WMF). The mariners need to select the best simulation result from each panel based on their own judgements. In the second part of the survey, the mariners are required to score the coastline echoes simulated by conventional methods and three fractal algorithms. Score scale is using a likert scale from 1 to 5 , where 1 is "bad" and 5 is "excellent". The evaluation results are summarized in Table 1. For example, comparing the coastline echo simulated by conventional method with the one by FBM, $30 \%$ of the mariners consider the conventional method is better and $70 \%$ of the mariners think otherwise. Regarding the evaluation scores, the median evaluation scores on the coastline echoes generated by conventional method and FBM are 3 points and 4 points respectively. In general, the results of the evaluation from experienced mariners validate the advantages of fractal algorithms in terms of simulation of coastline echoes.

\section{Performance analysis of improved fractal algorithms}

According to the time of scan line finishing a rotation in Table 2, the improved fractal algorithms can guarantee the scan line to rotate around in no more than three seconds regardless of whether the radar range is small (say $0.25 \mathrm{NM}$ ) or large (say $6 \mathrm{NM}$ ) or the number of iterations is many or few. By contrast, the unimproved fractal algorithms can result in significantly slower rotation of scan line or even simulation failure.

Moreover, the improved fractal algorithm can still keep a natural-looking pattern and detail of the coastline echoes. Taking an improved FBM algorithm with threshold value $(\mathrm{L})$ as an example, the coastline echoes generated by improved FBM algorithm (Fig. 10b and c) are still better representations of a natural coastline echo than the echoes generated by conventional method (Fig. 10a). Additionally, the natural pattern and Detail of the coastline echo improve as the threshold value (L) decreases.

\section{Comparison of three fractal algorithms}

Based on calculations under the same number of iterations (i) and amount of original sample points of a coastline, the total number of sample points used by a random Koch curve algorithm in the simulation process is $2^{i}$ times of those used by the FBM and WMF algorithm. Since the number of the sample points can directly affect the rotation speed of the scan line, the FBM and WMF algorithm performs better than the random Koch curve algorithm from this aspect.

In addition, the pattern of the coastline echoes generated by FBM algorithm can be adjusted by Hurst index $H$ conveniently. The pattern of the coastline echoes simulated by WMF algorithm can be adjusted by the different 


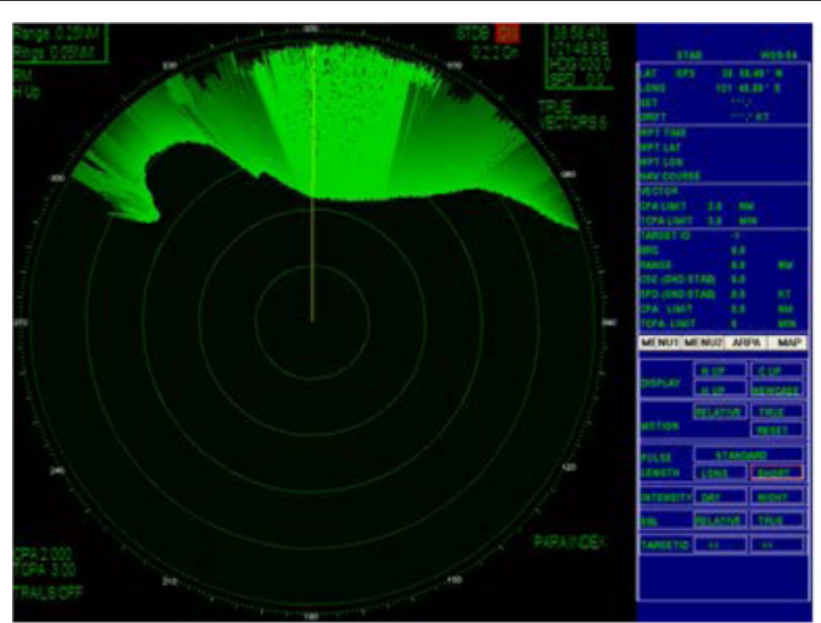

(a)

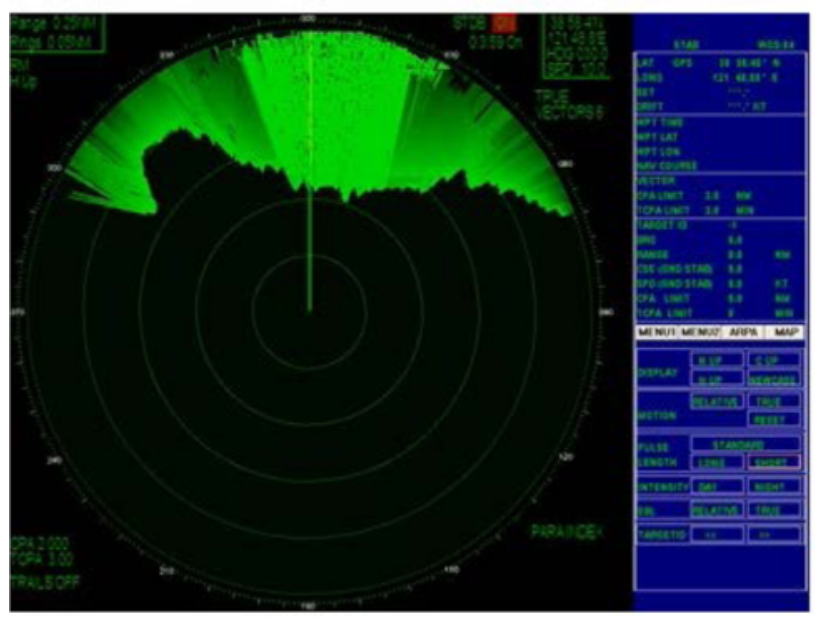

(c)

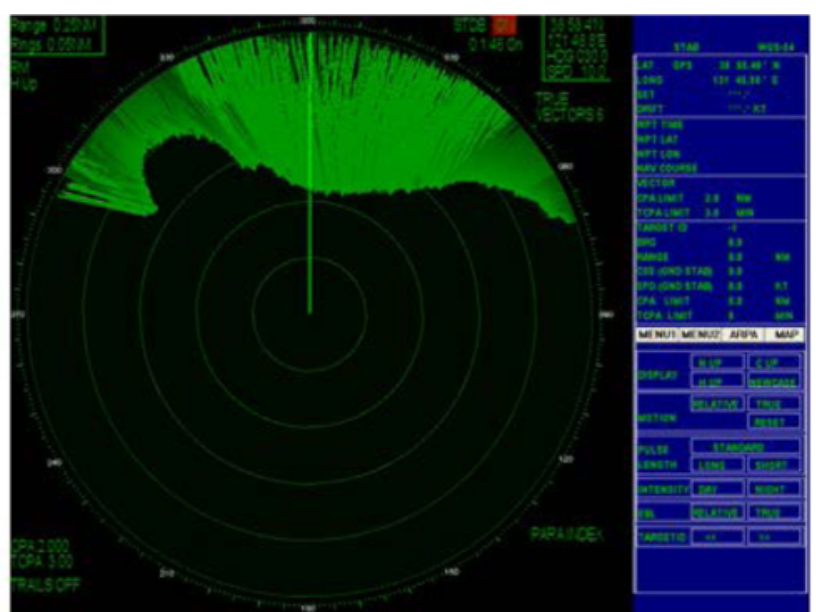

(b)

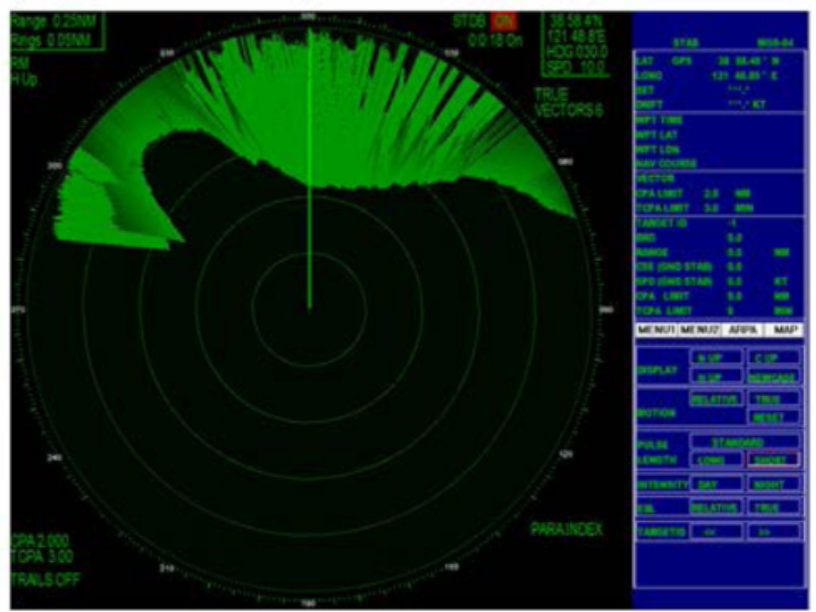

(d)

Fig. 9 Simulation outcomes of coastline echo. a by conventional method, b by random Koch curve algorithm, $\mathbf{c}$ by FBM algorithm, and $\mathbf{d}$ by WMF algorithm

combinations of $D, \gamma$, and $\phi_{n}$ as well. By contrast, the pattern of the coastline echoes generated by a random Koch curve algorithm can be changed very little. According to the simulation outcomes in this study, the coastline echoes generated by the FBM algorithm more closely

Table 1 Summary of evaluations from experience mariners on the simulated coastline echoes by various methods

\begin{tabular}{lll}
\hline Survey & Simulation method & Evaluation result \\
\hline Part I & Conventional Method v.s. Random Koch Curve & $30 \%$ v.s. $70 \%$ \\
& Conventional Method v.s. FBM & $30 \%$ v.s. $70 \%$ \\
& Conventional Method v.s. WMF & $33 \%$ v.s. $67 \%$ \\
Part II & Conventional Method & {$[2.00,3.00,4.00]$} \\
& Random Koch Curve & {$[3.00,3.00,4.00]$} \\
& FBM & {$[3.00,4.00,4.25]$} \\
WMF & {$[2.00,3.50,4.00]$}
\end{tabular}

Note: scores in square bracket are [25 \% quantile, median, $75 \%$ quantile]
Table 2 Speed of scan line to rotate a round by different algorithms, iterations, and radar ranges (unit in second/round)

\begin{tabular}{llllllllll}
\hline \multirow{2}{*}{ Algorithm } & Iteration & 6 & 5 & 4 & 3 & 2 & 1 & 0.5 & 0.25 \\
\hline Random Koch Curve & 1 & 3 & 3 & 3 & 3 & 3 & 3 & 3 & 3 \\
& 2 & 12 & 11 & 8 & 6 & 5 & 3 & 3 & 3 \\
& 3 & - & - & - & - & 8 & 7 & 6 & 5 \\
FBM & 1 & 3 & 3 & 3 & 3 & 3 & 3 & 3 & 3 \\
& 2 & 3 & 3 & 3 & 3 & 3 & 3 & 3 & 3 \\
& 3 & 5 & 4 & 3 & 3 & 3 & 3 & 3 & 3 \\
WMF & 4 & 12 & 11 & 8 & 6 & 5 & 5 & 5 & 3 \\
& 1 & 3 & 3 & 3 & 3 & 3 & 3 & 3 & 3 \\
Improved & 2 & 31 & 25 & 11 & 8 & 7 & 5 & 4 & 3 \\
Random Koch Curve/ & 2 & - & - & - & - & 14 & 11 & 8 & 6 \\
FBM/WMF & 3 & 3 & 3 & 3 & 3 & 3 & 3 & 3 & 3 \\
& 3 & 3 & 3 & 3 & 3 & 3 & 3 & 3 & 3 \\
& 4 & 3 & 3 & 3 & 3 & 3 & 3 & 3 & 3
\end{tabular}

Note: "-" means the simulation failed 


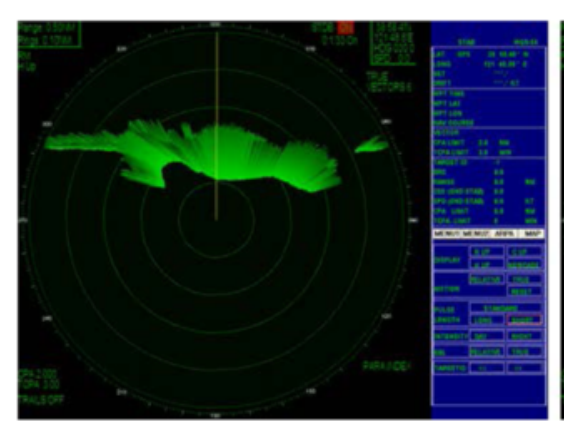

(a)

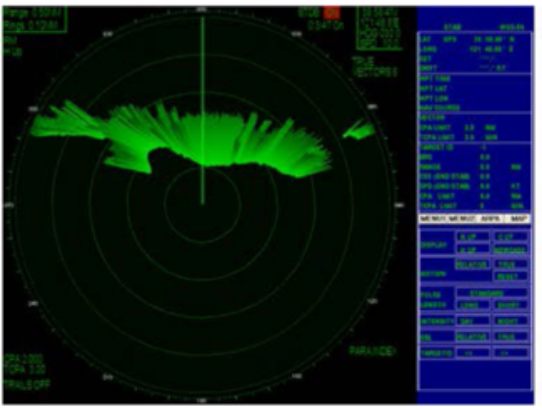

(b)

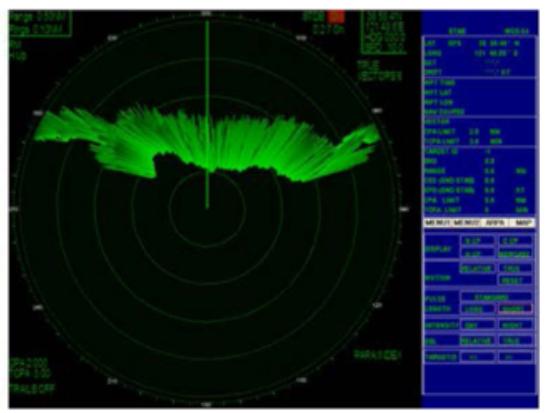

(c)

Fig. 10 Simulation outcomes of coastline echo. a by conventional method, $\mathbf{b}$ by improved FBM algorithm with $L=10$, and $\mathbf{c}$ by improved FBM algorithm with $L=3$

resemble real coastline echoes than the echoes generated by a random Koch curve and WMF algorithm.

\section{Conclusion}

In this paper, fractal algorithms are applied into the simulation of coastline echoes on marine radar simulator. The simulation outcomes from different methods are compared as well. In order to guarantee the rotating speed of radar scan line, threshold value $\mathrm{L}$ is introduced into the simulation process. Based on our evaluation, the improved FBM algorithm is the best choice for the simulation of coastline echoes on marine radar simulators. Natural-looking coastline echoes generated by the algorithms introduced in this study can improve the quality of the training significantly. The fractal algorithms developed in this paper are packaged into a dynamic link library (dll) with well documented application programmable interface (API), which means that the algorithms are decoupled from the simulator program. This brings great benefits and convenience when transplanting the algorithm to other simulators, as long as the simulator is able to load a dll library. Since a dll library is supported by most of Windows based programs, we believe that the adoption of our algorithms on other simulators will be effortless. One limitation of this study is that we only simulated Type II coastline echoes. Another limitation of this study is that we aim at applying the fractal algorithms into the simulation of the coastline echoes in radar simulator rather than finding the best parameters of the fractal algorithms for the simulation. Last but not least, since the authors are unable to collect real data from marine radar on board, a survey method is employed to evaluate the simulation results by fractal algorithm. In the future, simulation of other radar echo types will be considered; how to choose the parameters for the fractal algorithms should be investigated; and a more objective evaluation approach should be designed to evaluate the simulation results.

\section{Competing interests}

The authors declare that they have no competing interests.

\section{Authors' contributions}

SJ: coordinated the research, reviewed and analysed the literature, developed and implemented WMF algorithm, conducted survey and results analysis, and drafted the manuscript. ZZ: reviewed the literature, developed and implemented random Koch curve, FBM, and improvement algorithms, and drafted the manuscript. HY: developed and implemented WMF algorithm, designed survey questionaire, analyzed results, improved Koch curve and FBM algorithms, and revised the manuscript. DL: guided the development and implementation of random Koch curve and FBM algorithms, and contributed to visualization concept. RS: contributed to visualization concept and revised the manuscript. All authors read and approved the final manuscript.

\section{Author details \\ ${ }^{1}$ University of Tennessee, 37996 Knoxville TN, USA. ${ }^{2}$ Tangshan Maritime Safety Administration of People's Republic of China, 100736 Tangshan, China . \\ ${ }^{3}$ Department of Audio, Visual and Image Technology, National Police University of China, 100038 Shenyang, China.}

Received: 22 October 2015 Accepted: 10 March, 2016

Published online: 12 May 2016

\section{References}

Ali, A (2006). Simulator instructor-stcw requirements and reality. Pomorstvo: Scientific Journal of Maritime Research, 20(2), 23-32.

Arnold-Bos, A, Martin, A, Khenchaf, A (2006). A versatile bistatic polarimetric marine radar simulator, In Radar, 2006 IEEE Conference On (pp. 605-612).

Aviles, C, \& Scholz, C (1987). Fractal analysis applied to characteristic segments. Journal of Geophysical Research, 92(B1), 331-344

Baliarda, C.P, Romeu, J, Cardama, A (2000). The koch monopole: A small fractal antenna. Antennas and Propagation, IEEE Transactions on, 48(11), 1773-1781.

Berry, M, \& Lewis, Z (1980). On the weierstrass-mandelbrot fractal function, In Proceedings of the Royal Society of London A: Mathematical, Physical and Engineering Sciences, 370 (pp. 459-484): The Royal Society.

Boyle, R, Parvin, B, Koracin, D, Paragios, N, Tanveer, S.-M (2007). Advances in visual computing.

Bridge Command (2016). Interactive 3D Ship Simulator. https://www. bahookie.org/.

Cross, S.S (1994). The application of fractal geometric analysis to microscopic images. Micron, 25(1), 101-113.

Dalian Maritime University Institute of Navigational Technology (2016). Marine Radar Simulator. http://nvc.dlmu.edu.cn/list.php?fid=3.

Dieker, T (2004). Simulation of fractional brownian motion. The Netherlands: MSc theses, University of Twente, Amsterdam.

Falconer, K (2013). Fractals: A very short introduction. 
Filoche, M, \& Sapoval, B (2000). Transfer across random versus deterministic fractal interfaces. Physical review letters, 84(25), 5776.

Harrouni, S (2008). Fractal classification of typical meteorological days from global solar irradiance: application to five sites of different climates, 29-54.

Huang, S, Oelfke, S, Speck, R (1992). Applicability of fractal characterization and modelling to rock joint profiles, In International Journal of Rock Mechanics and Mining Sciences \& Geomechanics Abstracts, 29 (pp. 89-98): Elsevier.

Ji, S, Liu, D, Zhang, Z (2005). A review on fractal image compression coding and some improvement measures, In PDPTA'05 (pp. 151-156).

Ji, S, Zhang, Z, Yang, H, Liu, D, Sawhney, R (2015). Simulation of coastline's echo on marine radar simulator based on fractal theory, In Transportation Research Board 94th Annual Meeting.

Keddam, M, \& Takenouti, H (1988). Impedance of fractal interfaces: new data on the von koch model. Electrochimica acta, 33(3), 445-448.

Kongsberg Maritime (2016). Polaris Ships Bridge Simulator. http://www.km. kongsberg.com/ks/web/nokbg0240.nsf/AllWeb/ B2F29B3742D75297C1257315003C3F6F?OpenDocument.

Landfall (2016). Marine Radar Simulator. http://www.landfallnavigation.com/ radarsimulator.html.

Mandelbrot, B.B, \& Van Ness, J.W (1968). Fractional brownian motions, fractional noises and applications. SIAM review, 10(4), 422-437.

Mandelbrot, B (1979). Fractals: form, chance and dimension. Fractals: form, chance and dimension., by Mandelbrot, BB, 1, 16+ 365 .

Mandelbrot, B.B (1983). The fractal geometry of nature, 173.

Majumdar, A, \& Tien, C (1990). Fractal characterization and simulation of rough surfaces. Wear, 136(2), 313-327.

Ma, C, Yang, J, Zhao, L, Mei, X, Shi, H (2015). Simulation and experimental study on the thermally induced deformations of high-speed spindle system. Applied Thermal Engineering, 86, 251-268.

Nautical Software (2016). Marine Radar Simulator. http://www. nauticalsoftware.com/training-software/marine-radar-simulator.html.

Organization, I.M (2006). Internatinoal Convention on Standards of Training, Certification and Watchkeeping for Seafarers, 1978, as amended in 2006 London.

Pentland, A.P (1984). Fractal-based description of natural scenes. Pattern Analysis and Machine Intelligence, IEEE Transactions on (6), 661-674.

Rostek, S, \& Schöbel, R (2013). A note on the use of fractional brownian motion for financial modeling. Economic Modelling, 30, 30-35.

Shanhua, X, Songbo, R, Youde, W (2015). Three-dimensional surface parameters and multi-fractal spectrum of corroded steel. PloS one, 10(6), 0131361.

Teel, S, Sanders, J, Parrott, D.S, Wade, L, Gervais, T, Rovinski, K, Stone, L.C, Murai, K, Hayashi, Y (2009). Evaluation of marine simulator training based on heart rate variability, In Systems, Man and Cybernetics, 2009. SMC 2009. IEEE International Conference On (pp. 233-238): IEEE.

Voss, R.F (1988). Fractals in Nature: from Characterization to Simulation: Springer.

Wang, J, Wu, C, Liu, C, Wei, J (2015). Fractal simulation on random rough surface, In 2015 International Conference on Automation, Mechanical Control and Computational Engineering: Atlantis Press.

Weierstrass, K (1967). Über continuirliche funktionen eines reellen arguments, die für keinen werth des letzteren einen bestimmten differentialquotienten besitzen, 1872. Karl Weiertrass Mathematische Werke.

Xiuwen, L, Yong, Y, Yicheng, J, Xinyu, Z (2010). Design radar signal interface for navigation radar/arpa simulator using radar display, In Circuits, Communications and System (PACCS), 2010 Second Pacific-Asia Conference On, 1 (pp. 442-445): IEEE.

Yin, Y, Liu, X, Li, Z (2007). Key technologies of navigation radar simulator using real radar monitor. Journal of System Simulation, 19(5), 1014-1017.

Yang, X, Qin, K, Wu, C, Chen, L. Simulation of coastlines based on cloud fractal.

Zhang, Z, Liu, D, Han, Y, Ji, S (2005). Characteristics, Applications and the Prospects of DSP, In Parallel and Distributed Processing Techniques and Applications (pp. 161-165).

Zhang, Z (2007). Research On The Algorithm Of Generating Coastline Echo In Radar Based On Fractal Theory. China: Master's thesis, Dalian Maritime University.

Zhang, C, Zhang, D, Quan, D (2010). Echo image generation method for marine radar based on opengl modeling and simulation technology. Journal of Dalian Maritime University, 3, 017.

Zhang, L, Yu, C, Sun, J (2015). Generalized weierstrass-mandelbrot function model for actual stocks markets indexes with nonlinear characteristics. Fractals, 1550006.

\section{Submit your manuscript to a SpringerOpen ${ }^{\circ}$ journal and benefit from:}

- Convenient online submission

- Rigorous peer review

- Immediate publication on acceptance

- Open access: articles freely available online

- High visibility within the field

- Retaining the copyright to your article

Submit your next manuscript at $\mathbf{s p r i n g e r o p e n . c o m ~}$ 